\title{
Degradation of textile dyes using immobilized lignin peroxidase-like metalloporphines under mild experimental conditions
}

\author{
Paolo Zucca ${ }^{1,2^{*}}$, Antonio Rescigno ${ }^{1}$, Manuela Pintus ${ }^{1}$, Andrea C Rinaldi ${ }^{1}$ and Enrico Sanjust ${ }^{1}$
}

\begin{abstract}
Background: Synthetic dyes represent a broad and heterogeneous class of durable pollutants, that are released in large amounts by the textile industry. The ability of two immobilized metalloporphines (structurally emulating the ligninolytic peroxidases) to bleach six chosen dyes (alizarin red S, phenosafranine, xylenol orange, methylene blue, methyl green, and methyl orange) was compared to enzymatic catalysts. To achieve a green and sustainable process, very mild conditions were chosen.
\end{abstract}

Results: IPS/MnTSPP was the most promising biomimetic catalyst as it was able to effectively and quickly bleach all tested dyes. Biomimetic catalysis was fully characterized: maximum activity was centered at neutral $\mathrm{pH}$, in the absence of any organic solvent, using hydrogen peroxide as the oxidant. The immobilized metalloporphine kept a large part of its activity during multi-cycle use; however, well-known redox mediators were not able to increase its catalytic activity. IPS/MnTSPP was also more promising for use in industrial applications than its enzymatic counterparts (lignin peroxidase, laccase, manganese peroxidase, and horseradish peroxidase).

Conclusions: On the whole, the conditions were very mild (standard pressure, room temperature and neutral pH, using no organic solvents, and the most environmental-friendly oxidant) and a significant bleaching and partial mineralization of the dyes was achieved in approximately $1 \mathrm{~h}$. Therefore, the process was consistent with large-scale applications. The biomimetic catalyst also had more promising features than the enzymatic catalysts.

Keywords: Biomimetic, Dye, Lignin-peroxidase, Porphine, Porphyrin

\section{Background}

Textile dyes are a broad and heterogeneous class of molecules used in many technological fields (i.e., textile and paper production, food technology, and hair coloring) [1]. Many chemical classes of synthetic dyes are frequently employed on an industrial scale; azo dyes, triarylmethanes, phenothiazine, and anthraquinones are among the most widespread [2,3].

Because the goal of the dyeing process is color durability, textile dyes of all chemical classes are commonly highly resistant to both chemical and physical degradation. Accordingly, textile dyes represent a serious environmental concern because of their chemical inertness, high annual production (over 10,000 tons per year [1]),

\footnotetext{
* Correspondence: pzucca@unica.it

'Dipartimento di Scienze Biomediche, Università di Cagliari, Cittadella

Universitaria, 09042, Monserrato, CA, Italy

${ }^{2}$ Consorzio UNO (University of Oristano Consortium), Oristano, Italy
}

toxicity/carcinogenicity $[4,5]$, and the large amount of dyes released in industrial wastewaters (up to $50 \%$ of total process intake [6]).

The European Directive 2002/61/EC forbids the use of some products (in particular some azo dyes). However, these restrictions only marginally solve the environmental problem because of the large volume of other dyes discharged every year.

Several physical, chemical and enzymatic approaches have been proposed to remove dyes from industrial wastewaters [7-16] that require long reaction times, extreme operational conditions, and high costs [1]. Accordingly, the development of a clean and environmentally friendly process for removing dyes is a challenging issue, far from being solved.

Redox-active metalloporphines are efficient catalysts; in the presence of a proper oxidant [17-21], they are able to oxidize many substrates, including several textile dyes, 
under very mild conditions. We previously studied the ability of an immobilized manganese porphine to oxidize alizarin red S (ARS), an anthraquinone dye) [22], and phenosafranine (PNS), a phenylphenazinium dye [23], elucidating several aspects of the catalytic mechanism.

In this study, we compare the catalysts IPS/MnTSPP and PP-PVA/FeTFPP (Figure 1), each containing a metalloporphine (Mn- and Fe-, respectively) immobilized onto a solid support, thus emulating ligninolytic peroxidases [24]. We investigated the ability of these two catalysts to oxidize different chemical classes of textile dyes in the presence of hydrogen peroxide under mild conditions. We also report the results of these catalysts on ARS and PNS to allow a comprehensive comparison. All the dyes included in the study are reported in Table 1.

\section{Results and discussion}

\section{Comparison of IPS/MnTSPP and PP-PVA/FeTFPP}

We previously developed two biomimetic catalysts, IPS/ MnTSPP and PP-PVA/FeTFPP, which are able to oxidize several lignin-model compounds. IPS/MnTSPP strictly emulates a lignin peroxidase (LiP) active site, having an imidazole-grafted support that coordinates a $\mathrm{Mn}(\mathrm{III})$ porphine [24]. This catalyst showed a LiP-like activity, whereas, unlike an enzyme such as manganese peroxidase $(\mathrm{MnP})$, it was not able to use the $\mathrm{Mn}^{2+}$ ion as a redox mediator. Conversely, PP-PVA/FeTFPP was only "bioinspired" (i.e., only inspired by but not strictly emulating, a natural enzyme active site) because the metalloporphine was coordinated by a pyridine-grafted support [25] rather than imidazole, as in ligninolytic peroxidases. This catalyst showed both LiP-like and MnP-like catalytic activities.
These two catalysts were tested for their ability to decolorize several textile dyes in the presence and absence of $\mathrm{Mn}^{2+}$ using hydrogen peroxide as the oxidant in accordance with green chemistry principles [26]. The investigation employed very mild conditions (neutral $\mathrm{pH}$ and room temperature, using no organic solvents, and the most environmental-friendly oxidant) to serve as the basis for an environmentally sustainable textile wastewater treatment process.

The results of these tests are reported in Table 2 . Both biomimetic catalysts easily degraded all tested dyes, showing wide substrate specificity. As previously reported [24], the catalytic behavior of IPS/MnTSPP was not affected by the presence of $\mathrm{Mn}(\mathrm{II})$, showing similar bleaching percentages in both the presence and absence of this ion. All six dyes were rapidly bleached under the conditions studied; more than with a removal in all cases higher than $50 \%$ of each dye (up to > 90\% for PNS, MG, and MO) was removed in $1 \mathrm{~h}$. In contrast, PP-PVA/FeTFPP was almost inactive in the absence of a suitable redox mediator. The conversion of all six dyes increased in the presence of $\mathrm{Mn}^{2+}$, leading to values similar on average to those observed for IPS/MnTSPP.

Considering that textile wastewaters do not usually contain manganous ion and that it would therefore be necessary to add $\mathrm{Mn}^{2+}$, itself a heavy metal pollutant, to the reaction, IPS/MnTSPP was considered the most promising catalyst for future industrial applications Hence, the rest of study focused on IPS/MnTSPP.

The time dependence of dye bleaching by IPS/ MnTSPP is shown in Figure 2. Note that most of the bleaching occurs in the first hour of the reaction, consistent with industrial timing needs.
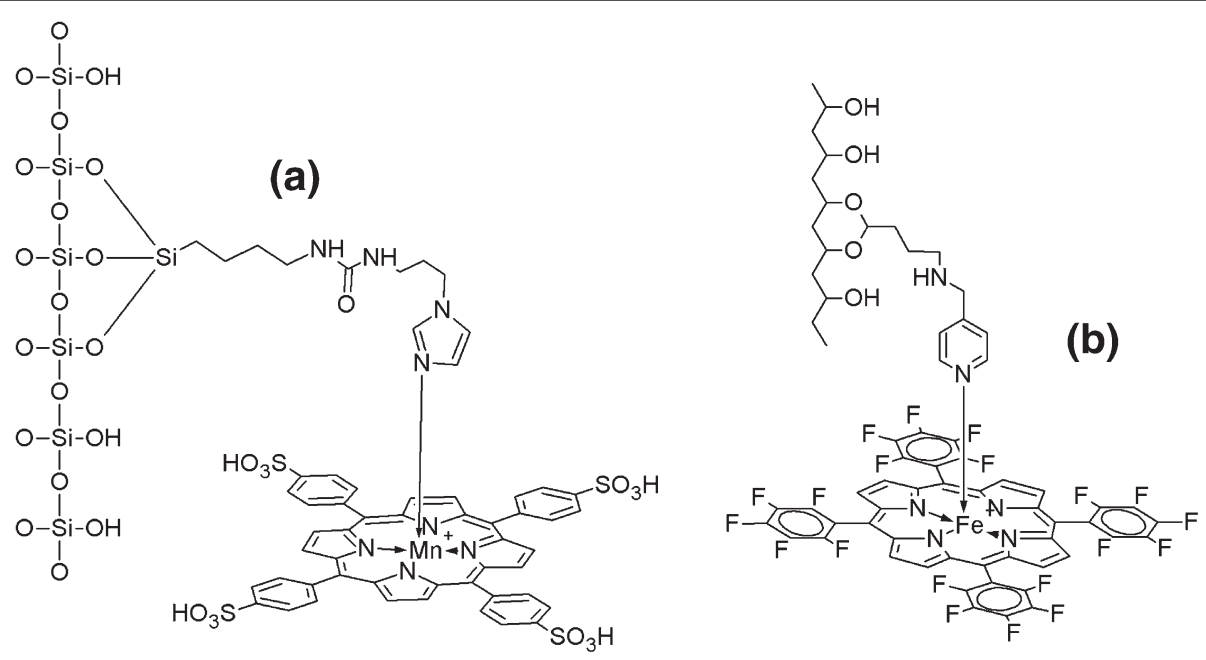

Figure 1 Proposed structure of the catalysts IPS/MnTSPP (a) and PP-PVA/FeTFPP (b). For more details, see: [24,25]. 
Table 1 The six textile dyes included in this study

\begin{tabular}{llccc}
\hline & DW (g/mol) & $\lambda_{\max }\left(\mathbf{n m}^{2}\right.$ & $\varepsilon\left(\mathrm{mol}^{*} \mathrm{~L}^{-1} * \mathrm{~cm}^{-1}\right)$ \\
\hline Alizarin Red S (ARS) & 342.26 & 520 \\
7200
\end{tabular}<smiles>Nc1ccc2nc3ccc(N)cc3[n+](-c3ccccc3)c2c1</smiles><smiles>CC1=CC(=C(c2cc(C)c(O)c(CN(CC(=O)[O-])CC(=O)[OH2+])c2)c2ccccc2S(=O)(=O)O)C=C(CN(CC(=O)[OH2+])CC(=O)[OH2+])C1=O</smiles><smiles>CN(C)c1ccc2nc3ccc(N(C)C)cc3[s+]c2c1</smiles><smiles>CC[N+](C)(C)c1ccc(C(=C2C=CC(=[N+](C)[O-])C=C2)c2ccc(N(C)C)cc2)cc1</smiles> 
Table 2 Removal of the studied dyes in the presence of hydrogen peroxide and either IPS/MnTSPP or PP-PVA/ FeTFPP, in both the presence and absence of $\mathrm{Mn}$ (II) as the redox mediator

\begin{tabular}{lccccccc}
\hline Mn(II) & Catalyst & \multicolumn{5}{c}{ Dyes removal \% (1 h) } \\
\cline { 3 - 8 } & & ARS & PNS & XO & MB & MG & MO \\
\hline \multirow{2}{*}{ No } & IPS/MnTSPP & $62 \pm 1$ & $92 \pm 2$ & $42 \pm 4$ & $96 \pm 4$ & $82 \pm 5$ & $87 \pm 2$ \\
\cline { 2 - 8 } & PP-PVA/FeTFPP & n.d. & n.d. & n.d. & n.d. & n.d. & n.d. \\
\hline \multirow{2}{*}{ Yes } & IPS/MnTSPP & $55 \pm 1$ & $88 \pm 2$ & $44 \pm 2$ & $91 \pm 3$ & $77 \pm 4$ & $85 \pm 4$ \\
\cline { 2 - 7 } & PP-PVA/FeTFPP & $48 \pm 3$ & $58 \pm 2$ & $55 \pm 5$ & $37 \pm 1$ & $48 \pm 2$ & $91 \pm 2$ \\
\hline
\end{tabular}

The reaction mixtures contained $5 \mathrm{mg}$ of catalyst in $1 \mathrm{~mL}$ of $25 \mathrm{mM}$ buffer solution containing $8.8 \mathrm{mM}$ hydrogen peroxide and a proper concentration of the selected dyes (0.29 mM ARS, $0.31 \mathrm{mM}$ PNS, $2 \mathrm{mM}$ XO, $0.15 \mathrm{mM} \mathrm{MB}$, $1.5 \mathrm{mM} \mathrm{MG}$, and $1.25 \mathrm{mM} \mathrm{MO}$ ). Quantification of the removed dye was obtained photometrically, using the $\lambda_{\max }$ and molar extinction coefficients reported in Table 1. n.d.: not detectable. $(n=4)$.

\section{Characterization of bleaching by IPS/MnTSPP and $\mathrm{H}_{2} \mathrm{O}_{2}$}

IPS/MnTSPP catalysis was strongly $\mathrm{pH}$-dependent; an approximately neutral $\mathrm{pH}$ was optimal for all dyes tested except XO and PNS, which had optimal bleaching at $\mathrm{pH}$ 6 and 8, respectively (data not shown). However, XO and PNS were sufficiently bleached at $\mathrm{pH} 7$ that IPS/ MnTSPP remains a viable catalyst for environmentally friendly wastewater treatment.

An exhaustive kinetic modeling of this catalysis is beyond the scope of our work. However, the decolorization of all six dyes apparently followed saturation MichaelisMenten-like kinetics, allowing the calculation of catalytic parameters (Table 3). Reported Michaelis constants $\left(\mathrm{K}_{\mathrm{M}}\right)$ showed that IPS/MnTSPP had significantly higher affinity for cationic dyes than anionic dyes (lower $K_{M}$ for

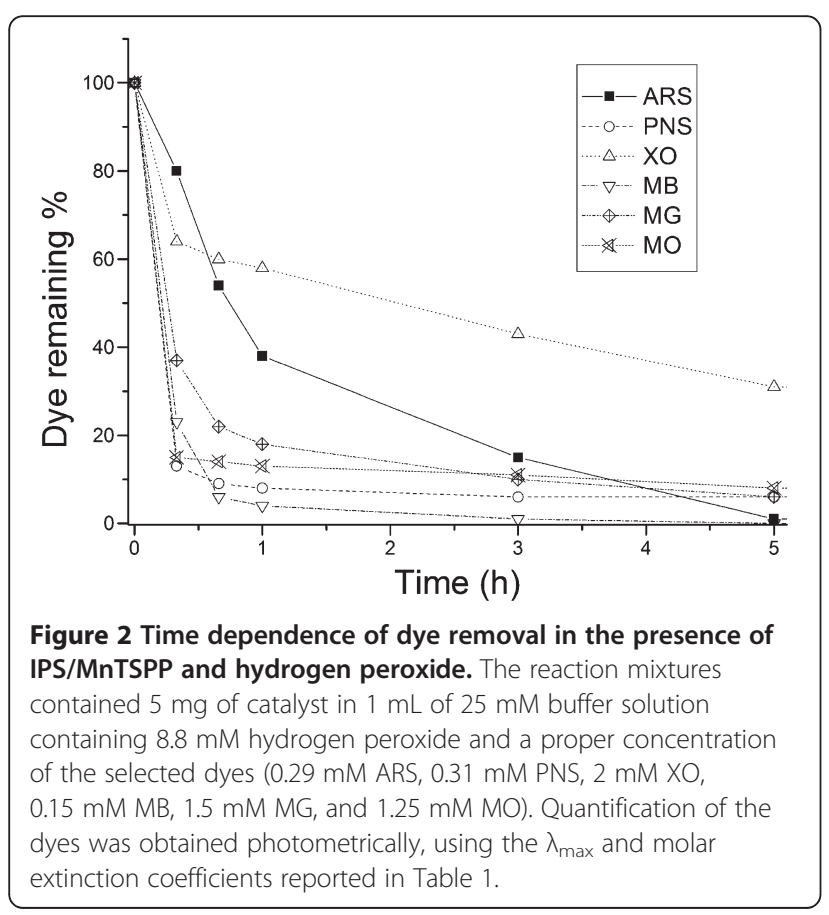

PNS and MB, higher $K_{M}$ for ARS and XO). This lower affinity led to the lowest bleaching rate for $\mathrm{XO}$ and ARS (compare Figure 2). Notably, in all cases, $K_{M}$ values were in the millimolar range and did not suffer from substrate inhibition. This can be a crucial issue for potential industrial applications because some bleaching approaches (i.e., enzymatic, vide ultra) are not able to work at these concentrations.

To test the hypothesis of a one-electron abstraction mechanism as the first step of the bleaching process, the influence of several well-known redox mediators was also studied: 4-hydroxy-2,2,6,6-tetramethylpiperidine-1oxyl (OH- TEMPO), $N$-hydroxyphthalimide (NPH), $N$ hydroxysuccinimide (NHS), and $N$-hydroxybenzotriazole (NHT). The results are summarized in Table 4. Only in the case of MG was an increase (three-fold) of the catalytic activity observed; in all the other cases, the redoxmediators mainly decreased IPS/MnTSPP catalysis (this quenching could be due to the lower reactivity of the oxygen-centered radical form of these mediators). Even in the best case, however, because the increase in catalytic activity was moderate, the industrial benefit of these redox mediators may not justify their environmental impact.

The catalyst was also investigated for its reuse potential. The results are reported in Table 5. On average, a rapid decrease of catalytic activity was observed in the first 2-3 cycles. Subsequently, activity was stable at approximately $25-30 \%$ of maximum for at least $8-10$ cycles. A potential explanation for this pattern is the possibility that a fraction of some dyes remains tightly adsorbed on the catalyst even when the supernatant is completely decolorized. This adsorbed dye would likely reduce the catalytic performance by steric hindrance and/or electrostatic repulsion of incoming substrate molecules. Nevertheless, the catalyst keeps a significant part of its catalytic activity during multi-cycle employment. This is an important quality for large-scale applications.

Significant removal of total organic carbon (TOC) was also observed during the bleaching by $\mathrm{H}_{2} \mathrm{O}_{2} / \mathrm{IPS} /$ MnTSPP, as shown in Figure 3. In the case of ARS, PNS, and $\mathrm{MB}$, over $50 \%$ of TOC removal was observed in 24 hours, whereas lower decreases were recorded for $\mathrm{XO}$ and MG. Conversely, in the case of MO no TOC modification occurred during the catalysis. These results suggest that a decarboxylative degradation occurred for some of the dyes, whose TOC values decreased significantly during the bleaching experiments.

IPS/MnTSPP was also tested for its ability to decolorize a complex mixture of all the six dyes, emulating realistic textile plant waste. The six dyes were each present in the saturating concentrations used above, leading to high impacting TOC (approximately $1580 \mathrm{mg} / \mathrm{L}$ ). The changes in the UV-vis spectra (Figure 4) show a 
Table 3 Kinetic parameters measured for the bleaching of each dye by IPS/MnTSPP and hydrogen peroxide

\begin{tabular}{|c|c|c|c|c|c|c|c|}
\hline \multirow[t]{2}{*}{ Sub-strate } & \multirow[t]{2}{*}{ Kinetic parameter } & \multicolumn{6}{|c|}{ Dye } \\
\hline & & ARS & PNS & XO & MB & MG & MO \\
\hline \multirow[t]{3}{*}{ Reducing substrate } & $\mathrm{K}_{M}(\mathrm{mM})$ & $2.11 \pm 0.74$ & $0.32 \pm 0.04$ & $1.33 \pm 0.38$ & $0.11 \pm 0.02$ & $0.72 \pm 0.12$ & $0.63 \pm 0.05$ \\
\hline & $k_{\text {cat }}\left(\min ^{-1}\right)$ & $0.14 \pm 0.03$ & $2.2 \pm 0.1$ & $98 \pm 14$ & $19.0 \pm 1.8$ & $108 \pm 9$ & $67 \pm 4$ \\
\hline & $\mathrm{k}_{\mathrm{cat}} / \mathrm{K}_{M}\left(\mathrm{mM}^{-1} \mathrm{~min}^{-1}\right)$ & $0.066 \pm 0.037$ & $7.0 \pm 1.2$ & $74 \pm 31$ & $166 \pm 52$ & $150 \pm 37$ & $105 \pm 16$ \\
\hline \multirow[t]{3}{*}{$\mathrm{H}_{2} \mathrm{O}_{2}$} & $\mathrm{~K}_{M}(\mathrm{mM})$ & $3.21 \pm 0.12$ & $3.0 \pm 0.4$ & $7.9 \pm 1.9$ & $2.4 \pm 0.5$ & $0.43 \pm 0.08$ & $7.4 \pm 1.5$ \\
\hline & $k_{\text {cat }}\left(\min ^{-1}\right)$ & $0.13 \pm 0.01$ & $1.9 \pm 0.1$ & $78 \pm 8.9$ & $15.2 \pm 1.3$ & $159 \pm 12$ & $71 \pm 8$ \\
\hline & $\overline{\mathrm{k}_{\mathrm{cat}} / \mathrm{K}_{M}\left(\mathrm{mM}^{-1} \mathrm{~min}^{-1}\right)}$ & $0.040 \pm 0.004$ & $0.63 \pm 0.13$ & $9.9 \pm 3.6$ & $6.4 \pm 2.1$ & $369 \pm 100$ & $9.6 \pm 3.2$ \\
\hline
\end{tabular}

Data for ARS and PNS were previously reported and are added here to allow a full comparison $[22,23](n=5)$.

significant decolorization, although with a slower kinetics (approximately 5 hours for a $50 \%$ decrease of the main visible peak). However, the feasibility of using this treatment in an industrial process was confirmed by a significant TOC decrease (approximately 20\%) in the same time frame.

\section{Comparison with enzymatic bleaching}

A full comparison with the enzymatic bleaching of the dyes was also performed. The ligninolytic enzymes tested were laccase (LC), lignin peroxidase, and the nonligninolytic enzyme horseradish peroxidase (HRP). In addition, $\mathrm{Mn}(\mathrm{III})$ was also used as oxidizing agent, mimicking manganese peroxidase action [27]. These biological catalysts suffered from significant substrate inhibition, preventing their use at the dye concentrations used for IPS/MnTSPP. Usually, concentrations of dyes at least of one order of magnitude lower were necessary. These enzymes also suffer from $\mathrm{H}_{2} \mathrm{O}_{2}$ inhibition, needing lower concentrations of oxidizing substrate. Accordingly, under the same conditions, it is not surprising that enzymatic bleaching efficiency was significantly lower than IPS/MnTSPP bleaching efficiency $(<1 \%$ dye removal in all cases). Enzymatic bleaching activity, under the conditions studied, is reported in Table 6, allowing a qualitative analysis of substrate specificity only.

None of the examined enzymes were able to bleach all the studied dyes. For instance, PNS is resistant to LC,

Table 4 Effect of well-known redox-mediators on the bleaching of the six tested dyes by IPS/MnTSPP

\begin{tabular}{ccccccc}
\hline \multirow{2}{*}{$\begin{array}{c}\text { Redox } \\
\text { mediator }\end{array}$} & \multicolumn{7}{c}{ \% Catalytic activity } \\
\cline { 2 - 7 } & ARS & PNS & XO & MB & MG & MO \\
\hline OH-TEMPO & 98 & 45 & 277 & 56 & 336 & 16 \\
\hline NHT & 142 & 46 & 162 & 38 & 299 & 121 \\
\hline NHS & 141 & 89 & 146 & 90 & 313 & 122 \\
\hline NPH & 103 & 32 & 74 & 56 & 340 & 57
\end{tabular}

Results are expressed by referring to a $100 \%$ reference activity in the absence of any mediator. Data for ARS and PNS were previously reported and are added here to allow a full comparison [22,23].
HRP, and $\mathrm{Mn}^{3}+$, whereas only a partial degradation with concomitant irreversible enzyme inactivation is observed with LiP. MB underwent only partial oxidation with the enzymatic catalysts.

IPS/MnTSPP is able to effectively bleach all the dyes studied, as described above. This feature, combined with its ability to oxidize relatively high concentration of dye (in the millimolar range), and with its partial multi-cycling, may make IPS/MnTSPP catalysis a feasible alternative for industrial treatment of textile wastewaters.

\section{Experimental section}

\section{Chemicals and instrumentation}

All reagents were the best commercial grade available and were used without further purification. In particular, silica gel 100 came from Fluka (cat. No. 60746), and MnTSPP and FeTFPP were purchased from SigmaAldrich (cat. No. 441813 and 252913, respectively), PVA from Aldrich (cat. No. 363138), glutaraldehyde from Fluka (cat. no. 49629), horseradish peroxidase (E.C. 1.11.1.7) and lignin peroxidase (E.C. 1.11.1.14) were from Sigma-Aldrich (cat No. P-6782 and 42603, respectively).

Table 5 Multi-cycle activity of IPS/MnTSPP

\begin{tabular}{ccccccc}
\hline $\begin{array}{c}\text { Catalytic } \\
\text { cycle }\end{array}$ & ARS & PNS & XO & MB & MG & MO \\
\cline { 2 - 7 } & 100 & 100 & 100 & 100 & 100 & 100 \\
\hline 1 & 68 & 73 & 100 & 99 & 98 & 44 \\
\hline 3 & 49 & 70 & 93 & 73 & 97 & 37 \\
\hline 4 & 33 & 61 & 88 & 49 & 97 & 34 \\
\hline 5 & 30 & 51 & 47 & 37 & 97 & 28 \\
\hline 6 & 28 & 49 & 41 & 34 & 96 & 28 \\
\hline 7 & 28 & 44 & 31 & 34 & 96 & 28 \\
\hline 8 & 26 & 41 & 30 & 26 & 94 & 27 \\
\hline 9 & 25 & 39 & 29 & 25 & 94 & 20 \\
\hline 10 & 25 & 35 & 27 & 24 & 90 & 19 \\
\hline
\end{tabular}

Catalytic activity of the IPS/MnTSPP adduct upon multicyclic use during the decolorization of the studied dyes $(n=3)$. Data for ARS and PNS were previously reported and are added here to allow a full comparison [22,23]. 


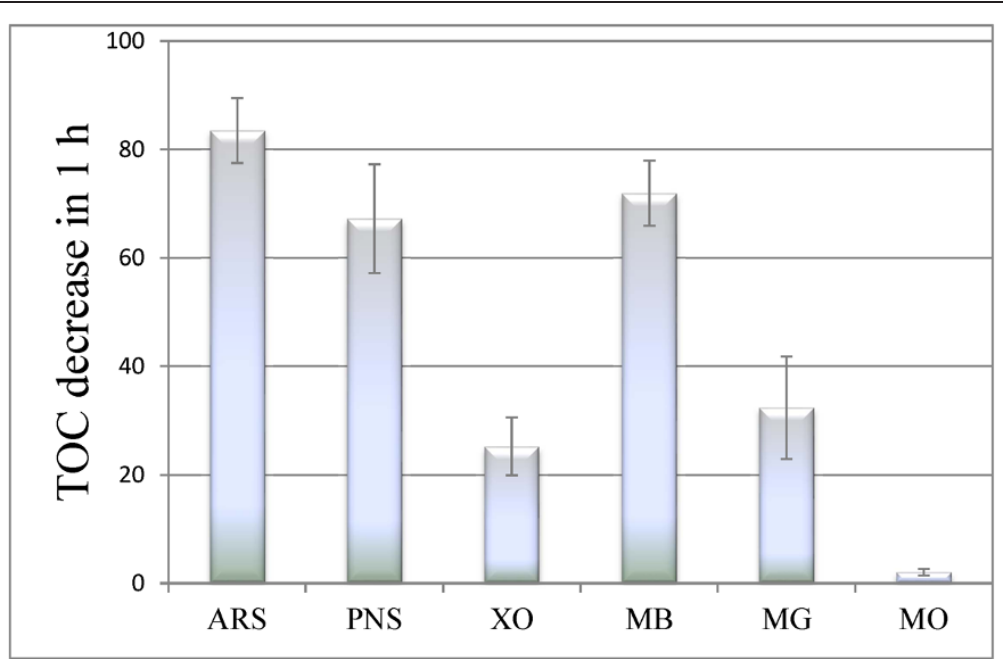

Figure 3 Total organic carbon (TOC) decrease during the bleaching of the textile dyes by IPS/MnTSPP $(n=3)$.

Laccase from Pleurotus sajor-caju was purified through two chromatographic steps (details in [28,29]).

Spectrophotometric measurements were carried out with an UltroSpec 2100pro (Amersham Bioscience). Concentrations of the substrates were also measured by UV-HPLC and GC-MS, using previously described methods $[30,31]$.

\section{Preparation of IPS/MnTSPP}

The complex IPS/MnTSPP was synthesized as previously described [24]. Briefly, $10 \mathrm{~g}$ plain silica gel was functionalized by overnight reaction at $80^{\circ} \mathrm{C}$ with 10 mmol 3-(1-imidazolyl)propylcarbamoyl-3'-aminopropyl- triethoxysilane solubilized in $10 \mathrm{~mL}$ dioxane, in the presence of $10 \mu \mathrm{L} \mathrm{N}$-methylmorpholine acting as the catalyst. The activated silica (IPS) was washed consecutively with $0.5 \mathrm{M} \mathrm{HCl}, \mathrm{H}_{2} \mathrm{O}, 0.1 \mathrm{M} \mathrm{NaOH}$ and $\mathrm{H}_{2} \mathrm{O}$ again. The wet silica was then carefully dried overnight in a vacuum oven.

The bound MnTSPP was quantified by the difference in absorption at $400 \mathrm{~nm}$ (the $\lambda_{\max }$ of the MnTSPP Soret band), as described in [24].

\section{Preparation of PP-PVA/FeTFPP}

The complex PP-PVA/FeTFPP was synthesized as previously described [25]. Briefly, $1 \mathrm{~g}$ of aminopropyl crosslinked

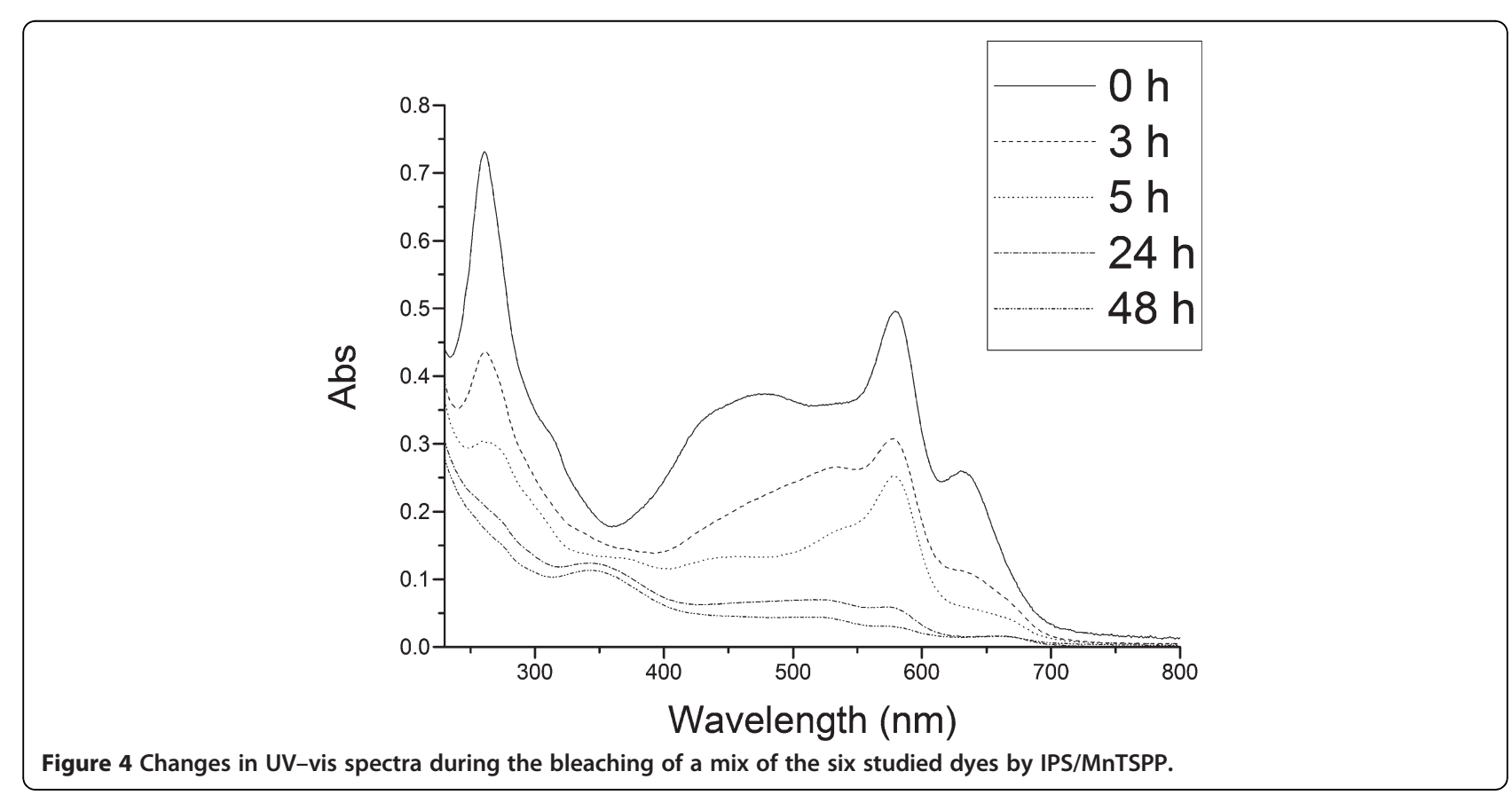


Table 6 Enzymatic bleaching of the dyes

\begin{tabular}{lccccc}
\hline Dye & $\mathrm{LC}$ & $\mathrm{LC}+\mathrm{H}_{2} \mathrm{O}_{2}$ & $\mathrm{HRP}$ & $\mathrm{LiP}$ & $\mathrm{Mn}^{3+}$ \\
\hline ARS & + & ++ & $+/-$ & $+/-$ & + \\
\hline PNS & - & - & - & $+/-$ & - \\
\hline XO & + & + & + & - & + \\
\hline MB & - & $+/-$ & $+/-$ & $+/-$ & - \\
\hline MG & + & + & ++ & $+/-$ & ++ \\
\hline MO & + & $+/-$ & + & $+/-$ & + \\
\hline
\end{tabular}

In the conditions tested, ligninolytic enzymes present a narrower substrate specificity towards the dyes included in this study than IPS/MnTSPP. Legend: LC laccase, HRP horseradish peroxidase, LiP lignin peroxidase, ++ good substrate (complete bleaching $<1 \mathrm{~h}$ ), + substrate, +/- weak substrate (bleaching $<10 \%$ in $24 \mathrm{~h}$ ), - no substrate.

PVA (prepared as in [32]) was suspended in an excess of $0.05 \mathrm{M}$ sodium acetate buffer $\mathrm{pH} 5$, and treated with $0.1 \mathrm{~mL}$ 4-pyridinecarboxaldehyde and $0.5 \mathrm{~g}$ sodium cyanoborohydride. After $24 \mathrm{~h}$ of incubation at $25^{\circ} \mathrm{C}$, the support was exhaustively washed with water, and dried at $50^{\circ} \mathrm{C}$, to obtain PP-PVA. One-gram aliquots of PP-PVA were treated with $20 \mathrm{mg}$ of FeTFPP, solubilized in $10 \mathrm{~mL}$ DMSO. The slurries were kept stirring in the dark for $24 \mathrm{~h}$, washed exhaustively with DMSO, and carefully dried at $50^{\circ} \mathrm{C}$.

Bound metalloporphine was quantified by difference through spectrophotometric measurement at $411 \mathrm{~nm}$ $\left(\varepsilon_{411}=115,000 \mathrm{M}^{-1} \mathrm{~cm}^{-1}\right.$ in DMSO $)$ [25].

\section{Activity measurements}

Routine measurements of the catalytic activity of immobilized metalloporphines were performed through spectrophotometric assays. The suspension contained $5 \mathrm{mg}$ of catalyst in $1 \mathrm{~mL}$ of $25 \mathrm{mM}$ buffer solution containing $8.8 \mathrm{mM}$ hydrogen peroxide and a proper concentration of the selected dye. The routine analyses were performed using the following concentrations of dyes (chosen to be only slightly higher than respective $K_{M}$, to better observe influences on catalytic activity): $0.29 \mathrm{mM}$ ARS, $0.31 \mathrm{mM}$ PNS, $2 \mathrm{mM} \mathrm{XO}, 0.15 \mathrm{mM} \mathrm{MB}, 1.5 \mathrm{mM} \mathrm{MG}$, and $1.25 \mathrm{mM}$ MO. During the kinetic experiments, wide ranges of substrate concentrations were studied (0.01-2 mM for the dyes, 0.1-40 $\mathrm{mM}$ for hydrogen peroxide).

The reaction mixture was then stirred at $25^{\circ} \mathrm{C}$ in the dark, and the reaction was monitored photometrically using the $\lambda_{\max }$ and molar extinction coefficients, reported in Table 1.

The actual hydrogen peroxide concentration was determined by $\mathrm{KMnO}_{4}$ titration.

When the reaction $\mathrm{pH}$ was not 7 , the $\mathrm{pH}$ was corrected to 7 with $0.1 \mathrm{~mL}$ of potassium phosphate buffer $1 \mathrm{M}$ ( $\mathrm{pH} 7$ ) before an absorbance measurement. UV-vis spectra in the range 230-700 $\mathrm{nm}$ were also recorded. Different Mcllvaine buffers were used in the $\mathrm{pH}$ range $3-8$.
When used, redox mediators (4-hydroxy-2,2,6,6-tetramethylpiperidine-1-oxyl $\mathrm{OH}$ - TEMPO; $N$-hydroxyphthalimide NPH; $N$-hydroxysuccinimide NHS; and $N$-hydroxybenzotriazole NHT) were added to the reaction medium at a final concentration of $1 \mathrm{mM}$. For the MnP-like assays, all the experiments were repeated as above in the presence of $1 \mathrm{mM} \mathrm{MnSO}_{4}$ and $50 \mathrm{mM}$ malonic acid. During multi-cyclic runs, the catalysts were regenerated between consecutive cycles by repeated washings with water and 2-propanol and by drying at $50^{\circ} \mathrm{C}$.

Total organic carbon determination was performed by automatic analyzer TOC 5000A (Shimadzu), equipped with $\mathrm{Pt}-\mathrm{Al}_{2} \mathrm{O}_{3}$ oxidizing catalyst. The volume of injection was 25 $\mu \mathrm{L}$. The calibration curve was obtained using a range of standard solutions of potassium phthalate (0-200 ppm).

Michaelis-Menten kinetic parameters were calculated by using R 2.5.1 software ( $R$ Foundation for Statistical Computing, Vienna).

\section{Enzymatic assays}

When horseradish peroxidase (HRP) was used, up to 20 E.U. were present in a final volume of $1 \mathrm{~mL}$ of $25 \mathrm{mM}$

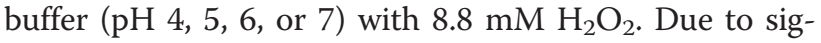
nificant substrate inhibition, lower dyes concentrations were used: $119 \mu \mathrm{M}$ ARS; $31 \mu \mathrm{M}$ PNS; $200 \mu \mathrm{M}$ XO; $15 \mu \mathrm{M}$ MB; $150 \mu \mathrm{M}$ MG; $65 \mu \mathrm{M}$ MO.

The same conditions were adopted for the experiments with laccase (23 E.U. added); every experiment was performed in the presence and absence of $\mathrm{H}_{2} \mathrm{O}_{2}$.

In the case of lignin peroxidase (LiP), 0.2 E.U. were present in the final volume of $1 \mathrm{~mL}$, with $0.176 \mathrm{mM}$ $\mathrm{H}_{2} \mathrm{O}_{2}$, due to strong substrate inhibition.

Moreover, to mimic manganese peroxidase ( $\mathrm{MnP})$ action, other experiments were conducted using $\mathrm{Mn}(\mathrm{III})$ as the putative oxidizing agent. One $\mathrm{mM}$ manganese triacetate was dissolved in $50 \mathrm{mM}$ sodium malonate buffer, $\mathrm{pH} 6.5$ [27], and the final mixture contained this solution along with dyes at the same concentrations, as mentioned above.

\section{Conclusion}

IPS/MnTSPP has proved able to efficiently degrade all tested dyes, regardless of their chemical class, under very mild conditions: standard pressure, room temperature and neutral $\mathrm{pH}$, using no organic solvents, and the most environmental-friendly oxidant. The yields and time requirements of the process were quite promising for a potential industrial application. Furthermore, a multicycle capability was described, even if only $30 \%$ of catalytic activity is retained for 8-10 cycles.

In comparison to enzymatic systems, the biomimetic catalyst was more efficient and had wider substrate specificity. Therefore, after proper optimization of the process, this catalyst is worth consideration as a possible medium/ large scale application in textile wastewater treatment. 


\section{Abbreviations}

ARS: Alizarin Red S; FeTFPP: 5,10,15,20-tetrakis(pentafluorophenyl) porphine-iron(III) chloride; IPS: (3-Imidazolylpropylcarbamoyl)-3'aminopropylsilica; HRP: Horseradish peroxidase; LC: Laccase; LiP: Lignin peroxidase; MB: Methylene Blue; MG: Methyl Green; MnTSPP: 5,10,15, 20-tetrakis(4-sulfonato-phenyl)porphine-Mn(III) chloride; MO: Methyl Orange; MnP: Manganese peroxidase; PP-PVA: 4'-Pyridylmethyl-3-aminopropylfunctionalized; PNS: Phenosafranine; PVA: Crosslinked with glutaraldehyde; TOC: Total organic carbon; XO: Xylenol Orange.

\section{Competing interests}

The authors declare that they have no competing interests.

\section{Authors' contributions}

PZ performed the main part of the experiments and drafted the manuscript. MP performed TOC and enzymatic assays. ACR, and AR helped analyzing the data and draft the manuscript. ES conceived the study, coordinated the study, and worked on data analysis and interpretation. All the authors read and approved the final manuscript.

\section{Acknowledgments}

This research was partly funded by Fondazione Banco di Sardegna (Project Code 861/2012) and Regione Autonoma Sardegna (PO Sardegna FSE 20072013, L.R.7/2007, Bando Giovani Ricercatori, Project CRP1_27, and PO Sardegna FSE 2007-2013, L.R.7/2007, Ricerca di Base Bando 2008, Project CRP1 408).

Received: 22 October 2012 Accepted: 19 December 2012 Published: 20 December 2012

\section{References}

1. Forgacs E, Cserhati T, Oros G: Removal of synthetic dyes from wastewaters: a review. Environ Int 2004, 30:953-971.

2. Azizi A, Moghaddam MRA, Arami M: Application of wood waste for removal of reactive blue 19 from aqueous solutions: optimization through response surface methodology. Environ Eng Manag J 2012, 11:795-804.

3. Brînzilă Cl, Ciobanu R, Brillas E: Effect of experimental parameters on crystal violet mineralization by electro-fenton processes. Environ Eng Manag J 2012, 11:517-523.

4. Tsuda S, Murakami M, Matsusaka N, Kano K, Taniguchi K, Sasaki YF: DNA Damage induced by red food dyes orally administered to pregnant and male mice. Toxicol Sci 2001, 61:92-99.

5. Walthall WK, Stark JD: The acute and chronic toxicity of two xanthene dyes, fluorescein sodium salt and phloxine B, to Daphnia pulex. Environ Pollut 1999, 104:207-215.

6. Pierce J: Colour in textile effluents - the origins of the problem. J Soc Dye Colour 1994, 110:131-133.

7. Colar LA, Ilinoiu EC, Manea F, Pode R, Lazau C: Photocatalytic activity of a silver doped TIO 2 modified zeolite in the degradation of reactive yellow 125 azo dye. Environ Eng Manag J 2012, 11:61-68.

8. Zucca P, Rescigno A, Olianas A, Maccioni S, Sollai F, Sanjust E: Induction, purification, and characterization of a laccase isozyme from Pleurotus sajor-caju and the potential in decolorization of textile dyes. J Mol Catal B Enzym 2011, 68:216-222

9. Pakshirajan K, Jaiswal S, Das RK: Biodecolourization of azo dyes using phanerochaete chrysosporium: effect of culture conditions and enzyme activities. J Sci Ind Res 2011, 70:987-991.

10. Córdoba A, Magario I, Ferreira ML: Experimental design and MM2-PM6 molecular modelling of hematin as a peroxidase-like catalyst in Alizarin Red S degradation. J Mol Catal A Chem 2012, 355:44-60.

11. Zhu L, Ghosh T, Park CY, Meng ZD, Oh WC: Enhanced sonocatalytic degradation of rhodamine B by graphene-TiO 2 composites synthesized by an ultrasonic-assisted method. Chin J Catal 2012, 33:1276-1283.

12. Oh WC, Chen M, Cho K, Kim C, Meng Z, Zhu L: Synthesis of graphene-CdSe composite by a simple hydrothermal method and its photocatalytic degradation of organic dyes. Chin J Catal 2011, 32:1577-1583.

13. Asgher M, Kamal S, lqbal HMN: Improvement of catalytic efficiency, thermostability and Dye decolorization capability of pleurotus ostreatus IBL-02 laccase by hydrophobic Sol Gel entrapment. Chem Centr J 2012, 110

14. Stengl V, Matys Grygar T, Henych J, Kormunda M: Hydrogen peroxide route to Sn-doped titania photocatalysts. Chem Centr J 2012, 113.
15. Velmurugan $\mathrm{R}$, Sreedhar B, Swaminathan M: Nanostructured $\mathrm{AgBr}$ loaded TiO 2: an efficient sunlight active photocatalyst for degradation of reactive Red 120. Chem Centr J 2011, 5

16. Chiavola A: Textiles. Water Environ Res 2012, 84:1511-1532.

17. Zucca P, Rescigno A, Sanjust E: Ligninolytic peroxidase-like activity of a synthetic metalloporphine immobilized onto mercapto-grafted crosslinked PVA inspired by the active site of cytochrome P450. Chin J Catal 2011, 32:1663-1666.

18. Faria AL, Mac Leod TOC, Barros VP, Assis MD: Hydrocarbon oxidation catalyzed by iron and manganese porphyrins anchored on aminofunctionalized supports. J Brazil Chem Soc 2009, 20:895-906.

19. Dos Santos JS, Faria AL, Da Silva Amorin PM, La Luna FM, Caiado KL, Silva DOC E, Sartoratto PPC, Assis MD: Iron(III) porphyrin covalently supported onto magnetic amino-functionalized nanospheres as catalyst for hydrocarbon and herbicide oxidations. J Brazil Chem Soc 2012, 23:1411-1420

20. Díaz-Díaz G, Blanco-López MC, Lobo-Castañón MJ, Miranda-Ordieres AJ, Tuñón-Blanco P: Hemo-acrylic polymers as catalyst for the oxidative dehalogenation of 2,4,6-trichlorophenol. Chloroperoxidase's mimic imprinting effects. J Mol Catal A Chem 2012, 353-354:117-121.

21. Tian P, Gao B, Chen $Y$ : Synchronous synthesis and immobilization of pyridyl porphyrins on crosslinked polystyrene microspheres and catalytic oxidation performance of immobilized cationic Co-porphyrins. Chin J Catal 2011, 32:483-489.

22. Zucca P, Vinci C, Sollai F, Rescigno A, Sanjust E: Degradation of Alizarin Red $\mathrm{S}$ under mild experimental conditions by immobilized 5,10,15,20tetrakis(4-sulfonatophenyl)porphine-Mn(III) as a biomimetic peroxidaselike catalyst. J Mol Catal A Chem 2008, 288:97-102.

23. Zucca P, Vinci C, Rescigno A, Dumitriu E, Sanjust E: Is the bleaching of phenosafranine by hydrogen peroxide oxidation catalyzed by silicasupported 5,10,15,20-tetrakis-(sulfonatophenyl)porphine-Mn(III) really biomimetic? J Mol Catal A Chem 2010, 321:27-33.

24. Zucca P, Mocci G, Rescigno A, Sanjust E: 5,10,15,20-Tetrakis(4-sulfonatophenyl)porphine-Mn(III) immobilized on imidazole activacted silica as a novel lignin-peroxidase-like biomimetic catalyst. J Mol Catal A Chem 2007, 278:220-227.

25. Zucca P, Sollai F, Garau A, Rescigno A, Sanjust E: Fe(III)-5,10,15,20-Tetrakis (pentafluorophenyl)porphine supported on pyridyl-functionalized, crosslinked poly(vinylalcohol) as a biomimetic versatile-peroxidase-like catalyst. J Mol Catal A Chem 2009, 306:89-96.

26. Cojocariu AM, Mutin PH, Dumitriu E, Vioux A, Fajula F, Hulea V: Removal of dimethylsulfoxide from wastewater using mild oxidation with $\mathrm{H}_{2} \mathrm{O}_{2}$ over Ti-based catalysts. Chemosphere 2009, 77:1065-1068.

27. Wariishi H, Valli K, Gold MH: Manganese(II) oxidation by manganese peroxidase from the basidiomycete phanerochaete-chrysosporium - kinetic mechanism and role of chelators. J Biol Chem 1992, 267:23688-23695.

28. Rescigno A, Zucca P, Flurkey A, Inlow J, Flurkey WH: Identification and discrimination between some contaminant enzyme activities in commercial preparations of mushroom tyrosinase. Enzyme Microb Technol 2007, 41:620-627.

29. Sollai F, Zucca P, Sanjust E, Steri D, Rescigno A: Umbelliferone and esculetin: inhibitors or substrates for polyphenol oxidases? Biol Pharm Bull 2008, 31:2187-2193.

30. Zucca P, Littarru M, Rescigno A, Sanjust E: Cofactor recycling for selective enzymatic biotrasformation of cinnamaldehyde to cinnamyl alcohol. Biosci Biotechnol Biochem 2009, 73:1224-1226.

31. Rescigno A, Casañola-Martin GM, Sanjust E, Zucca P, Marrero-Ponce Y: Vanilloid derivatives as tyrosinase inhibitors driven by virtual screeningbased QSAR models. Drug Test Anal 2011, 3:176-181.

32. Sanjust E, Cocco D, Curreli N, Rescigno A, Sollai F, Bannister JV: Flavingrafted poly(vinyl alcohol): preparation and properties. J Appl Polym Sci 2002, 85:2471-2477.

doi:10.1186/1752-153X-6-161

Cite this article as: Zucca et al:: Degradation of textile dyes using immobilized lignin peroxidase-like metalloporphines under mild experimental conditions. Chemistry Central Journal 2012 6:161. 